\title{
Brain Connectivity Models for Functional Magnetic Resonance Imaging
}

\author{
Nicole Geissberger ${ }^{1,2^{*}}$, Günter Schneckenreither ${ }^{2}$ \\ ${ }^{1}$ Center for Medical Physics and Biomedical Engineering, Medical University of Vienna, Währinger Gürtel 18-20, \\ 1090 Vienna, Austria; ${ }^{*}$ nicole.geissberger@tmsfmri.com \\ ${ }^{2}$ Institute for Analysis and Scientific Computing, TU Wien, Wiedner Hauptstraße 8-10, 1040 Vienna, Austria
}

SNE 27(3), 2017, 161-166, DOI: 10.11128/sne.27.tn.10387

Received: July 10, 2017; Revised: August 15, 2017;

Accepted: August 30, 2017

SNE - Simulation Notes Europe, ARGESIM Publisher Vienna, ISSN Print 2305-9974, Online 2306-0271, www.sne-journal.org

Abstract. When analyzing data from functional magnetic resonance imaging, different mathematical models are used. This article briefly describes the most important ones - starting with the so-called balloon model describing the hemodynamic response during brain activity, the General Linear Model widely used for functional localization of brain areas involved with certain stimuli, and Dynamic Causal Modelling as a framework for investigating dynamic brain connectivity models.

\section{Introduction}

Functional magnetic resonance imaging (fMRI) is a neuroimaging technique for investigating the inner mechanisms of the human brain during neuronal activity. A sequence of 3D images is acquired concurrently to stimuli to detect subtle changes in brain metabolism. Applications range from brain mapping - i.e. functional localization of brain regions associated with effects of interest - via research on brain connectivity to multimodal integration with other imaging and stimulation tools.

\section{Balloon Model}

The principle of fMRI is based on changes in cerebral blood supply. Neuronal activity causes an increased oxygen demand, therefore this demand must be met with an increase in inflow of highly oxygenated blood, overcompensating the energy demand.
Blood oxygenation increases, more specifically deoxygenated hemoglobin is reduced. Since deoxygenated hemoglobin $(\mathrm{dHb})$ is more paramagnetic than oxygenated hemoglobin $(\mathrm{oHb})$, magnetic field variations around $\mathrm{dHb}$ show reduced MR signal amplitudes. Thus, neuronal activity leads to a local decrease in $\mathrm{dHb}$ - causing an increased MR signal [5], [8] with a certain delay of several seconds.

This non-linear behaviour of the MR signal due to changes in blood supply is called the Blood Oxygenation Level Dependent (BOLD) effect. This is modelled using the so-called Balloon Model, linking changes in blood oxygenation caused by neuronal activity to the stimulus-induced hemodynamic response.
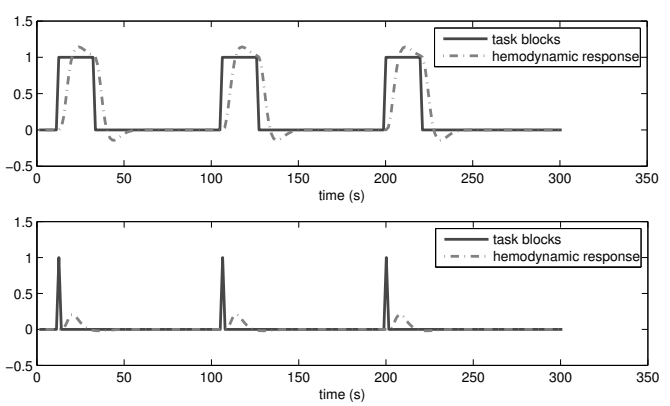

Figure 1: Hemodynamic response function (HRF) corresponding to task blocks with duration of 20 seconds (above) or events (below). Data created using the SPM software package.

The Balloon Model can be described by the following four differential equations, describing the signal $s$, the inflow of blood $f_{\text {in }}$, blood volume $v$, and deoxyhemoglobin $q$. It is assumed that the flow inducing signal $s$ and the change in regional cerebral blood flow $f_{\text {in }}$ are linked linearly,

$$
\dot{f}_{\text {in }}=s
$$


The flow inducing signal is generated by neuronal response to the stimulus function $u(t)$,

$$
\dot{s}=\varepsilon u(t)-\kappa s-\gamma\left(f_{\text {in }}-1\right)
$$

with the parameter $\varepsilon$ describing the efficacy of the stimulus to signal increase, $\kappa$ and $\gamma$ being rate constants describing signal decay and autoregulatory feedback from blood flow. The venous blood volume is the difference between inflow $f_{\text {in }}$ and outflow $f_{\text {out }}$ within a certain time interval (transit time) $\tau$,

$$
\dot{v}=\frac{f_{\text {in }}-f_{\text {out }}}{\tau} .
$$

The blood vessels show higher outflow rate when distended, modelled using a single stiffness parameter $\alpha$ as

$$
f_{\text {out }}=v^{\frac{1}{\alpha}}
$$

This describes the dynamic flow-volume relationship based on the so-called Windkessel model (see [6]). The deoxyhemoglobin content $q$ is modelled as an effect of blood flow and volume change,

$$
\begin{aligned}
\dot{q} & =\left(f_{\text {in }} \frac{E\left(f_{\text {in }}, \rho\right)}{\rho}-f_{\text {out }}(v) \frac{q}{v}\right) \frac{1}{\tau}, \\
E\left(f_{\text {in }}, \rho\right) & =1-(1-\rho)^{1 / f_{\text {in }}},
\end{aligned}
$$

with $E\left(f_{\text {in }}, \rho\right)$ describing the oxygen extraction from the inflowing arterial blood, and $\rho$ being the constant oxygen extraction fraction.

The six unknown biophysical parameters are thus: the stimulus efficacy $\varepsilon$, the rate constant for signal decay $\kappa$, the rate constant for blood flow autoregulation $\gamma$, the stiffness parameter $\alpha$, the resting oxygen extraction fraction $\rho$ and the mean transit time $\tau$. Commonly used estimates for these parameters can be seen in [2].

Assuming these six parameters to be constant, this allows to view the model described by these differential equations as a single input single output model - the input being the stimulus function $u(t)$ and the output being the measured BOLD signal $x(t)$. It can be written as

$$
x(t)=H(u(t))
$$

with $H$ being the hemodynamic response function (HRF), translating the stimulus $u(t)$ into the measured signal change $x(t)$. In practice, the time courses of the observed data and stimulus function are represented as vectors, thus can be written as

$$
\mathbf{x}=H(\mathbf{u})
$$

\section{General Linear Model (GLM)}

The General Linear Model is one of the most versatile models used in statistics. Many common statistical approaches, such as analysis of variance (ANOVA), ttests, ordinary linear regression etc. are special cases of this model [11].

For a series of measurements $\mathbf{y}$ and a matrix of explanatory variables - the so-called design matrix, the General Linear Model is described as

$$
\mathbf{y}=\mathbf{X} \beta+\varepsilon .
$$

Here, $\beta$ describes the parameters for modelling the linear coherence between the influencing factors and the output signal; $\varepsilon$ is the residual vector containing errors or noise, which are assumed independent and identically distributed.

\subsection{Embedding the Balloon Model in GLM}

Since for fMRI data the signal is not measured directly but rather through the hemodynamic response, this behaviour must be taken into account. The Balloon Model is incorporated into the design matrix as follows:

$$
\begin{aligned}
\mathbf{X} & =H(\mathbf{U}) \\
\mathbf{y} & =\mathbf{X} \beta+\varepsilon .
\end{aligned}
$$

For simplicity and because hemodynamic parameters can be assumed to be constant [2], the hemodynamic response function is usually not mentioned explicitly. The transformation of the stimulus functions in the design matrix is obtained by convoluting the stimulus function with the HRF.

\subsection{Application}

In functional MRI data 3D images are taken at every time point, resulting in a time course for each voxel. The coefficients $\beta$ are estimated minimizing the error squares. This Least Squares estimation can be easily performed using the so-called pseudoinverse of the design matrix:

$$
\beta \approx\left(\mathbf{X}^{\top} \mathbf{X}\right)^{-1} \mathbf{X}^{\top} \mathbf{y}
$$


To test for significance of the estimated $\beta$, the residual term $\mathbf{r}$ needs to be examined. It is defined as the difference between the measured values and the estimation,

$$
\mathbf{r}=\mathbf{y}-\mathbf{X} \beta .
$$

The standard deviation $\hat{\sigma}$ of the error term is estimated as

$$
\hat{\sigma}=\frac{\mathbf{r}^{\top} \cdot \mathbf{r}}{v}
$$

where $v$ is the number of degrees of freedom associated with $\mathbf{r}$. For independently distributed residuals in fMRI, this would be the number of scans minus the number of effects estimated (column rank of $\mathbf{X}$ ). The standard error $e$ can be described as

$$
e^{2}=\mathbf{c} \cdot \hat{\sigma}\left(\mathbf{X}^{\top} \mathbf{X}\right)^{-1} \cdot \mathbf{c}^{\top} .
$$

Now the t-score is obtained as

$$
t=\frac{\mathbf{c} \cdot \beta}{e} .
$$

The General Linear Model is estimated separately for each voxel, resulting in a 3D map of parameter estimates and corresponding t-scores which can be used to functionally localize brain activity associated with certain stimuli or tasks.

The described methods for functional neuroimaging data are implemented in the SPM package for MATLAB, which was developed by the Wellcome Trust Centre for Neuroimaging at the University College London. The software, as well as documentation and course files, is freely available at http://www.fil.ion.ucl. ac.uk/spm/software/.

\subsection{Criticism}

The GLM gives a robust and easy-to-use method for fMRI analyses, as no prior knowledge of function or activation maps are required to do a standard GLM analysis. However, it is limited to regarding just snapshots of brain states, neglecting brain dynamics and interplay of different cortical regions. Therefore, other methods must be used for more complex questions concerning interconnections and co-dependencies of brain regions.

\section{Dynamic Causal Modelling (DCM)}

Dynamic Causal Modelling is a framework which can be used for investigating hypotheses on effective brain connectivity, modelling the network behaviour of certain well-defined brain regions. This is done using following differential equation,

$$
\dot{\mathbf{z}}=F\left(\mathbf{z}, u, \theta_{c}\right)=\left(\mathbf{A}+\sum_{j} u_{j} \mathbf{B}^{j}\right) \mathbf{z}+\mathbf{C} u
$$

where $\mathbf{z}$ is the state vector describing the activity in a number of neuronal populations, $u$ is the (timedependent) input, the parameter $\theta_{c}$ describes the (timeindependent) coupling parameters, consisting of following adjacency matrices.

Here $\mathbf{A}$ describes the static influence of activity in each neuronal population on others independent of conditions (task/input-independent effective connectivity), $\mathbf{B}^{j}$ describes the condition-dependent intermodulations for each condition $j$, and $\mathbf{C}$ describes the direct influence of the conditions on activity in each brain region. The indices of the matrix entries specify the direction of the modulations - the column index indicating the source and the row index indicating the target of the connection.

Thus, the modeller needs to decide which regions of interest should be included in the model, as well as which connections are hypothesized to be present or not. The connection strength (i.e. the values of the entries in the connectivity matrices) is subsequently estimated from the data.

An example of such structural hypotheses in a DCM with 3 neuronal populations is depicted in figure 2 . Here, the assumed static connectivity is shown in blue, non-existing connections are indicated in red, influence of the conditions are shown in green. This DCM is translated into following $\mathbf{A}, \mathbf{B}$ and $\mathbf{C}$ matrices:

$$
\begin{array}{r}
{\left[\begin{array}{c}
\dot{z_{1}} \\
\dot{z_{2}} \\
\dot{z_{3}}
\end{array}\right]=\left(\left[\begin{array}{ccc}
a_{1,1} & a_{1,2} & 0 \\
a_{2,1} & a_{2,2} & a_{2,3} \\
0 & a_{3,2} & a_{3,3}
\end{array}\right]+u_{1}\left[\begin{array}{ccc}
0 & b_{1,2} & 0 \\
0 & 0 & 0 \\
0 & 0 & 0
\end{array}\right]\right)\left[\begin{array}{l}
z_{1} \\
z_{2} \\
z_{3}
\end{array}\right]} \\
+\left[\begin{array}{cc}
a_{1} & 0 \\
0 & 0 \\
0 & 0
\end{array}\right]\left[\begin{array}{l}
u_{1} \\
u_{2}
\end{array}\right]
\end{array}
$$




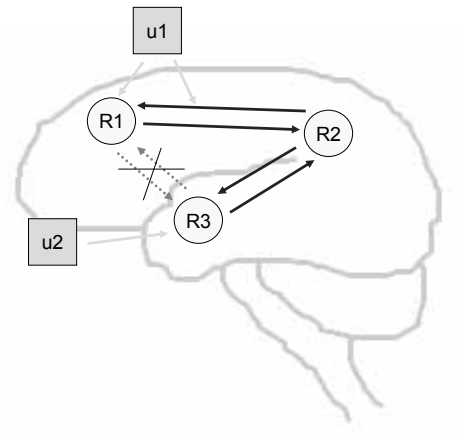

Figure 2: Schematic image of DCM structural assumptions with 3 neuronal populations.

\subsection{Embedding the Balloon Model in DCM}

As before, neuronal activity is not measured directly but via the BOLD effect - thus the hemodynamic state equations in section 1 need to be incorporated into the model as well. This leads to following full model from stimulus $u(t)$ to measured signal $y(t)$ :

stimulus functions

$$
\begin{gathered}
u(t) \\
\downarrow \\
\text { neural state equation } \\
\frac{d x}{d t}=\left(A+\sum_{j=1}^{m} u_{j} B^{j}\right) x+C u \\
\downarrow
\end{gathered}
$$

hemodynamic state equations

$$
\begin{gathered}
\dot{s}=x-\kappa s-\gamma(f-1) \\
\dot{f}=s \\
\tau \dot{v}=f_{\text {in }}-v^{\frac{1}{\alpha}} \\
\tau \dot{q}=f_{\text {in }} \frac{E\left(f_{\text {in }}, \rho\right)}{\rho}-v^{\frac{1}{\alpha}} \frac{q}{v} \\
\downarrow
\end{gathered}
$$

BOLD signal change equation

$$
y=\lambda(v, q)
$$

The BOLD signal change is modelled as a function of blood volume $v$ and deoxyhemoglobin content $q$,

$$
\lambda(v, q)=V_{0}\left(k_{1}(1-q)+k_{2}(1-q / v)+k_{3}(1-v)\right)
$$

taking into account the blood volume fraction $V_{0}$ and constants $k_{1}, k_{2}$ and $k_{3}$. These constants have been estimated by Buxton et al. [1] for a field strength of 1.5 Tesla. The variables of interest lying within the neural state equation, they can be estimated using a model inversion framework.

\subsection{Application}

Current implementations of DCM [4] within the SPM package use a Bayesian framework for model estimation, iteratively updating the parameters based on prior estimates to maximize the model evidence $p(y \mid m)$,

$$
p(y \mid m)=\int p(y \mid \theta, m) p(\theta \mid m) d \theta
$$

where $y$ is the measured data and $m$ is the model.

Maximizing the model evidence. As the model evidence (likelihood) can not be evaluated analytically, it needs to be approximated. Usually the maximization task is performed based on the log-likelihood due to numerical advantages when reaching very low values (for $p(y \mid m) \ll 1)$.

To reduce the risk of overfitting, state-of-the-art methods for model evidence estimations, such as the Bayesian Information Criterion (BIC) or the Akaike Information Criterion (AIC), generally use an accuracy and a complexity term to find the best balance between model fit and complexity.

$$
\log p(y \mid m) \approx \operatorname{accuracy}(m)-\operatorname{complexity}(m) .
$$

These criteria are defined as

$$
\begin{array}{r}
-\mathrm{AIC}=\log p(y \mid \theta, m)-n \\
-\mathrm{BIC}=\log p(y \mid \theta, m)-\frac{n}{2} \log (N)
\end{array}
$$

where $y$ stands for the data, $m$ is the model with parameters $\theta, N$ is the number of data points and $n$ is the number of parameters. Here, the complexity term penalizes a high number of parameters, however not their information content. Therefore, the so-called negative free energy was introduced,

$$
F=\langle\log p(y \mid \theta, m)\rangle_{q}-\mathrm{KL}[q(\boldsymbol{\theta}), p(\boldsymbol{\theta} \mid m)]
$$


where $q$ is the assumed conditional distribution, thus the left term is the expected log-likelihood under this posterior. KL denotes the Kullberg-Leibler divergence, which takes into account not only the number of parameters, but also their co-dependencies and therefore information gain,

$$
\begin{array}{r}
\mathrm{KL}[q(\theta), p(\theta \mid m)]=\frac{1}{2}\left(\log \left|C_{\theta}\right|-\log \left|C_{\theta \mid y}\right|\right. \\
\left.+\left(\mu_{\theta \mid y}-\mu_{\theta}\right)^{T} C_{\theta}^{-1}\left(\mu_{\theta \mid y}-\mu_{\theta}\right)\right) .
\end{array}
$$

Here, $C_{\theta}$ is the empirical prior covariance, $C_{\theta \mid y}$ is the posterior covariance, $\mu_{\theta}$ and $\mu_{\theta \mid y}$ are the prior and posterior expectation. The negative free energy is a lower bound for the log-likelihood and can thus be used as its approximation for parameter estimation and making single subject and group inferences on model structure.

Note that these measures - AIC, BIC as well as negative free energy - are relative measures only. Thus, they can be used to compare relative fit of different models, however not for testing overall model quality in the sense of hypothesis testing.

Parameter estimation. In the Dynamic Causal Modelling framework, both biophysical $\left(\theta_{h}=(\varepsilon, \kappa, \gamma, \alpha, \rho, \tau)\right.$, see section 1$)$ and connectivity parameters $\left(\theta_{c}=(A, B, C)\right)$ need to be estimated. As a non-linear optimization problem, current implementations of DCM use a Gauss-Newton algorithm in an Expectation Maximization Scheme (see [4]) to calculate the maximum a-posteriori (MAP) estimates. Here, a fully Bayesian approach is used, based on the conditional probability of the parameters given the data, $p(\theta \mid y)$.

According to Bayes' theorem, following proportionality is given:

$$
p(\theta \mid y) \propto p(y \mid \theta) p(\theta)
$$

Under Gaussian assumptions for the posterior density $p(\theta \mid y)$, its estimation is reduced to finding its first two moments - the conditional mean $\eta_{\theta \mid y}$ and covariance $C_{\theta \mid y}$ of $\theta \mid y$. Analogously, the priors are estimated in terms of their expectation $\eta_{\theta}$ and $C_{\theta}$.

From equation (24) we receive

$$
p(\theta \mid y) \propto \exp \left[-\frac{1}{2}\left(\theta-\eta_{\theta \mid y}^{(i+1)}\right)^{\top} C_{\theta \mid y}^{-1}\left(\theta-\eta_{\theta \mid y}^{(i+1)}\right)\right]
$$

with

$$
\begin{aligned}
C_{\theta \mid y} & =\left(\bar{J}^{\top} \bar{C}_{e}^{-1} \bar{J}\right)^{-1}, \\
\eta_{\theta \mid y}^{(i+1)} & =\eta_{\theta \mid y}^{(i)}+C_{\theta \mid y}\left(\bar{J}^{\top} \bar{C}_{e}^{-1} \bar{J}\right)
\end{aligned}
$$

where

$$
\bar{y}=\left[\begin{array}{c}
y-h\left(\eta_{\theta \mid y}^{(i)}\right) \\
\eta_{\theta}-\eta_{\theta \mid y}^{(i)}
\end{array}\right], \bar{J}=\left[\begin{array}{l}
J \\
1
\end{array}\right], \bar{C}_{e}=\left[\begin{array}{cc}
C_{e} & 0 \\
0 & C_{\theta}
\end{array}\right] .
$$

Iterating equation (25) when no priors are given can be seen as the Gauss-Newton method for parameter estimation (see [3] for further details).

Although we have so far assumed the error covariance $C_{e}$ to be known, usually it is not, e.g. for temporal correlations in fMRI. However, it can be estimated using some hyperparameters $\lambda_{j}$, so that

$$
\begin{aligned}
C_{e} & =\sum_{j} \lambda_{j} Q_{j} \quad \text { and } \\
Q_{j} & =\frac{\partial C_{e}}{\partial \lambda_{j}} .
\end{aligned}
$$

$Q_{j}$ represents a covariance basis set that embody variance components, which can model different variances for different data blocks, or even temporal correlations within blocks. To model the error covariance using this basis set, we must now estimate its coefficients $\lambda_{j}$.

The posterior mean $\eta_{\theta \mid y}$ and covariance $C_{\theta \mid y}$ as well as error covariance $C_{e}$ are now estimated iteratively in an expectation maximization (EM) scheme, such that the model evidence - i.e. the negative free energy $F-$ is maximized. See [3] and [7] for more details.

Inference strategies. After specification and estimation of a DCM, for each connectivity parameter in $\theta_{c}$ there exists an estimate of the mean and covariance, as well as an estimate of the model evidence through the negative free energy $F$.

On the parameter level, the significance of each connection can be tested using one-sided t-tests on the mean and covariance - analogously to section 2.2.

However, as the negative free energy is only a relative measure of model quality, we can not infer on the model itself, but only compare different models (model structures) on the same data, giving following options for inferences on the model structure level:

- Select the model maximizing the model evidence for each subject. 
- Average over models showing high posterior probability.

The first option is also referred to as Bayesian Model Selection (BMS). As there are often several models showing non-neglectable posterior probability, the second option has become an often preferred choice - referred to as Bayesian Model Averaging (BMA) - performing a weighted average over models within an Occam's window, i.e. showing a posterior probability above a certain threshold, see [9] and [10].

\subsection{Criticism}

Compared to classic GLM approaches, DCM inherently takes into account the dynamic nature of the brain by implementing interconnections between brain regions. However, the so-called "effective connectivity" investigated in the DCM does not imply direct physical connection of certain brain regions.

Also, while in the GLM analysis no prior assumptions have to be made on spatial extent and locations of activation changes, DCM depends strongly on a-priori hypotheses regarding neuronal populations and mechanisms.

Exploratory, i.e. non-hypothesis-driven analysis, could theoretically be conducted by setting each measurement point, i.e. voxel time course, in relation to each other and variating the existing connections respectively. However, this is not only numerically impossible, but also the winning model might not be optimal.

Within the current DCM framework it is only possible to estimate the most plausible parameters for predefined modulatory connections, but it is not possible to validate the prior assumptions (i.e. presumed connections) per se, and thus the resulting model. Thus, the DCM framework can only be regarded as a model selection framework. Other, possibly invasive, methods must be used for finding further evidence on the validity of the models.

\section{Acknowledgement}

We thank Christian Windischberger and his team at the Medical University of Vienna for sharing their knowledge and experience throughout this work.

\section{References}

[1] Buxton RB, Wong EC, and Frank, LR. Dynamics of blood flow and oxygenation changes during brain activation: the balloon model. Magnetic Resonance in Medicine. 1998; 39(6):855-864.

[2] Friston KJ, Mechelli A, Turner R, and Price CJ. Nonlinear responses in fMRI: the balloon model, volterra kernels, and other hemodynamics. NeuroImage. 2000; 12(4):466-477.

[3] Friston KJ. Bayesian estimation of dynamical systems: an application to fMRI. NeuroImage. 2002; 16(2):513-530.

[4] Friston K, Harrison L, and Penny W. Dynamic Causal Modelling. NeuroImage. 2003; 19:1273-1302.

[5] Logothetis NK, Pausl J, Augath M, Trinath T, and Oeltermann A. Neurophysiological investigation of the basis of the fMRI signal. Nature. 2001; 412(6843):150-157.

[6] Mandeville JB, Marota JJ, Ayata C, Zaharchuk G, Moskowitz MA, Rosen BR, and Weiskoff RM. Evidence of a cerebrovascular postarteriole windkessel with delayed compliance. Journal of Cerebral Blood Flow \& Metabolism. 1999; 19(6):679-689.

[7] Neal RM and Hinton GE. A view of the EM algorithm that justifies incremental, sparse, and other variants. In: Jordan MI. Learning in Graphical Models. First edition. Dordrecht: Springer Netherlands; 1998. pp 355-368.

[8] Ogawa S, Lee T-M, Kay AR, and Tank DW. Brain magnetic resonance imaging with contrast dependent on blood oxygenation. Proceedings of the National Academy of Sciences. 1990; 87(24):9868-9872.

[9] Penny WD, Stephan KE, Mechelli A and Friston KJ. Comparing dynamic causal models. NeuroImage. 2004; 22(3):1157-1172.

[10] Penny WD, Stephan KE, Daunizeau J, Rosa MJ, Friston KJ, Schofield TM and Leff AP. Comparing families of dynamic causal models. PLoS Comput Biol. 2010; 6(3):e1000709.

[11] Sachs L. Angewandte Statistik: Anwendung Statistischer Methoden. Eighth edition. Berlin Heidelberg: Springer-Verlag; 1997. 885 p. 